\title{
Research
}

\section{Methodological problems in dementia research in primary care: a case study of a randomized controlled trial}

\begin{abstract}
Jane Wilcock Department of Primary Care and Population Sciences, RFUCLMS, London, UK, Michelle Bryans Department of Clinical and Counselling Psychology, NHS Lanarkshire, East Kilbride, UK, Stephen Turner Dental Health Services Research Unit, Dundee, Scotland, UK, Ronan O'Carroll Department of Psychology, University of Stirling, Stirling, Scotland, UK, John Keady Northumbria University/for Dementia, School of Health, Community and Education Studies, Northumbria University, Benton, Newcastle-upon-Tyne, UK, Enid Levin Social Care Institute for Excellence, London, UK, Steve Iliffe Department of Primary Care and Population Sciences, National Co-ordinating Centre for Neurodegenerative Diseases and Dementias Research Networks, RFUCLMS, London, UK and Murna Downs Bradford Dementia Group, School of Health Studies, University of Bradford, Bradford, UK
\end{abstract}

The emerging UK national research network in neurodegenerative diseases and dementias aims to promote large-scale community-based studies of therapeutic interventions, based in primary care. However, trials in primary care settings can be problematic, a common difficulty being the recruitment of a large enough sample. The article discusses recruitment issues in a multi-centre randomized controlled trial of differing educational approaches to improving dementia care in general practice. Sample size calculations based on community studies of prevalence may be misleading in intervention trials which may recruit practices with atypical demography. Recruitment rates for practitioners in this study were lower than expected. Professionals excluded themselves from the study mainly due to pressures of time and staff shortages, and we detected both ambivalent attitudes to primary care research and a perception that research into dementia care was not a high priority. Evaluation of the quality of care may be perceived as criticism of clinical practice, at a time when general practice is undergoing major administrative and contractual changes. Variations in Research Ethics Committee conditions for approval led to different methods of recruitment of patients and carers into the study, a factor which may have contributed to disparate levels of recruitment across study sites. Patient and carer levels of recruitment were lower than expected and were affected partly by carers' time pressures and other family commitments, but largely by problems in identifying patients and carers in the practices. The development of research potential in primary care is at an early stage and studies reliant on patient recruitment in general practice must allow for multiple obstacles to enrollment. This is particularly relevant for studies of dementia care, where the prevalence of dementia in a demographically average population is low and the incidence very low, compared with other disabilities. Professionals may give dementia low priority in allocating practice time for research projects, and strategies to address this problem are needed.

Key words: dementia; primary care; recruitment; research methods

Received: January 2006; accepted: August 2006

Address for correspondence: Jane Wilcock, Department of Primary Care and Population Sciences, RFUCLMS, Rowland Hill St., London NW3 2PF, UK. Email: j.wilcock@pcps.ucl.ac.uk

\section{Background}

The newly formed UK National Co-ordinating Centre for Neurodegenerative disease and 
dementias research networks aims to develop local networks that can recruit large numbers of people for community-based trials. Primary care researchers will have an important role in developing these networks, and carrying out the trials (Department of Health, 2005). The need for more research to be carried out within primary care, and for primary care practitioners to develop their research capacity, is now well established (Mant, 1997). Furthermore, more research is needed on dementia in primary care, on new diagnostic tools and therapeutic interventions, and on the use of clinical signs and symptoms to estimate prognosis and select the clinical strategies which are utilized (Woods et al., 2003). As in other areas of health service research, a particular set of challenges faces the researcher in dementia care. Some issues arise from the subject under investigation and others derive from the context, including the working environments of primary care.

In this article we describe problems encountered in conducting a randomized controlled trial (RCT) of dementia education methods within a primary care setting (Downs et al., 2006). Our study started from the premise that the primary care team is generally the first point of contact for people with dementia and their families. Despite this pivotal role, there is evidence that general practitioners (GPs) often fail to detect dementia or to provide families with sufficient information about the illness or available support (Fortinsky et al., 1995; Downs, 1996; Iliffe, 1997; Boise et al., 1999; Audit Commission, 2000, 2002).

We accepted the paradigm that the RCT was the optimum method of answering our research questions (Cochrane, 1972; Haines and Iliffe, 1995). The validity of RCTs is dependent on obtaining and retaining sufficient numbers of participants both at the practice and patient level. Obtaining an adequate sample size can be problematic within primary care, and some studies have reported recruitment rates as low as one in ten of the potential sample (Thomas, 2000; Wilson et al., 2000).

\section{Methods}

\section{Setting}

The aim of the study was to test the differential efficacy of three educational interventions on practitioner knowledge, clinical practice and on outcomes for carers, using a mixed methodology approach to evaluation of complex educational interventions (Abbasi, 1999). It uses a pre-test post-test randomized trial design in which 35 primary care practices were randomly assigned to one of four interventions (see Figure 1). Details of the study design are reported elsewhere (Iliffe et al., 2002a), as are the educational interventions (Wilcock et al., 2002; Iliffe et al., 2002b; Turner et al., 2003). The study took place in Lothian and Forth Valley Health Board areas in Scotland and in Camden \& Islington, and Barnet Health Authority areas in London during 2000-2002. Since the study aimed to link medical records to patients and their carers, and to measure changes in clinical care and carer satisfaction after educational interventions at practice level, people with dementia had to be recruited through general practice.

\section{Sample size calculations}

The study set recruitment targets of 40 practices and we anticipated that up to 160 GPs and approximately 40 practice nurses would be recruited. We assumed that the 160 GPs in the study would generate 400 'cases' at baseline and that 600 one year following the intervention. We expected to contact

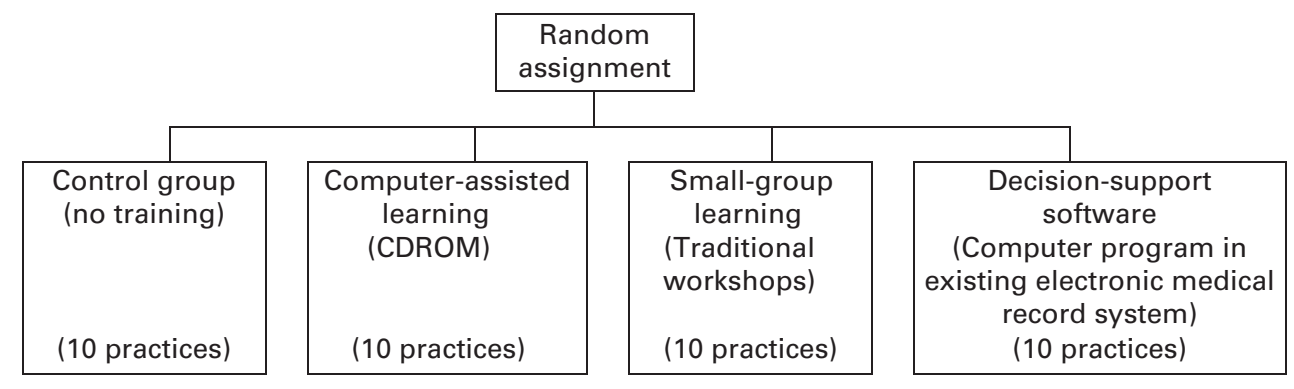

Figure 1 Study design 
Table 1 Study domains, variables and associated outcome measures gathered pre- and post-intervention

\begin{tabular}{|c|c|c|}
\hline Domain & Variables & Data sources \\
\hline Rates of diagnosis & $\begin{array}{l}\text { Diagnosis of dementia, or } \\
\text { possible dementia }\end{array}$ & $\begin{array}{l}\text { Search of electronic and paper medical } \\
\text { records for new diagnoses, conducted } \\
\text { by practice staff }\end{array}$ \\
\hline $\begin{array}{l}\text { Professionals' concordance } \\
\text { with clinical Guidelines }\end{array}$ & $\begin{array}{l}\text { Diagnostic and management in } \\
\text { clinical practice }\end{array}$ & $\begin{array}{l}\text { Search of electronic and paper } \\
\text { medical records }\end{array}$ \\
\hline $\begin{array}{l}\text { Professionals' knowledge } \\
\text { about content of clinical } \\
\text { guidelines }\end{array}$ & $\begin{array}{l}\text { Dementia knowledge } \\
\text { Perception of dementia-related } \\
\text { personal practice (caseload etc) }\end{array}$ & $\begin{array}{l}\text { Dementia Quiz } \\
\text { Practitioner questionnaire }\end{array}$ \\
\hline $\begin{array}{l}\text { Carers' knowledge about } \\
\text { dementia and use of services }\end{array}$ & $\begin{array}{l}\text { Dementia knowledge } \\
\text { Awareness of available services }\end{array}$ & $\begin{array}{l}\text { Dementia Quiz } \\
\text { Semi-structured Interview }\end{array}$ \\
\hline
\end{tabular}

10 live-in family carers per practice (200 per area) at baseline and 15 per practice one year following the intervention.

\section{Data capture}

The effectiveness of the interventions was assessed through change in a range of process and outcome measures relating to practices and to individual GPs, practice nurses, and informal carers. Table 1 summarizes the domains of the study and the variables and measures associated with each domain. The data capture tools were designed to have the minimum impact on practice staff workload. Practitioners, practice managers and carers were involved in the design and piloting process. Each measure was taken twice over a period of one year.

\section{Ethical committee requirements}

Applications were made to the local research ethical committees in the first five months of the three-year study.

\section{Practice recruitment strategy}

The study was designed to recruit a range of practice sizes in different geographic and demographic areas. The aim was to include a mix of urban and rural practices, previous practice experience of research and non-research, training and non-training practices, and a range of socioeconomically different areas. The steps involved in recruitment of practices are shown in Figure 2.

Practice inclusion criteria were: a computerized practice with (1) software amenable to introduction Primary Health Care Research and Development 2007; 8: 12-21 of decision support sub-programmes; (2) space for small group work; (3) access to at least one PC with a Windows operating system.

Each practice was given financial compensation in the form of a training grant for time spent on paperwork and in training and postgraduate training credits. Locum costs to backfill clinicians' time used in training were also offered, if desired.

All practices in the two London Health Authority areas were invited by letter from a grant holder to join the study, and this was followed by a phone call by research staff, initially to the practice manager. Clinical governance leads at each of the primary care groups in London were informed of the study by letter and asked to assist with aiding practice involvement.

If interest was shown a practice visit was arranged to discuss the details of the project, where possible as part of one of the regular clinical meetings at each practice. The team was not asked for an agreement to participate whilst the research team were present but were given time to reach an agreement in private. Over the course of eleven months nine London practices were recruited in this way.

Eleven practices were still required to ensure the target sample size was reached. A newsletter was distributed along with a covering letter stressing that recruitment was ongoing. Articles were also published in local research and educational bulletins. Several London practices responded to this and were followed up with a telephone call and a request for a practice visit. An additional eight London practices were recruited in this way.

In Scotland, the process of recruitment reflected the different organizational structure of primary care. First, presentations were made at meetings of 


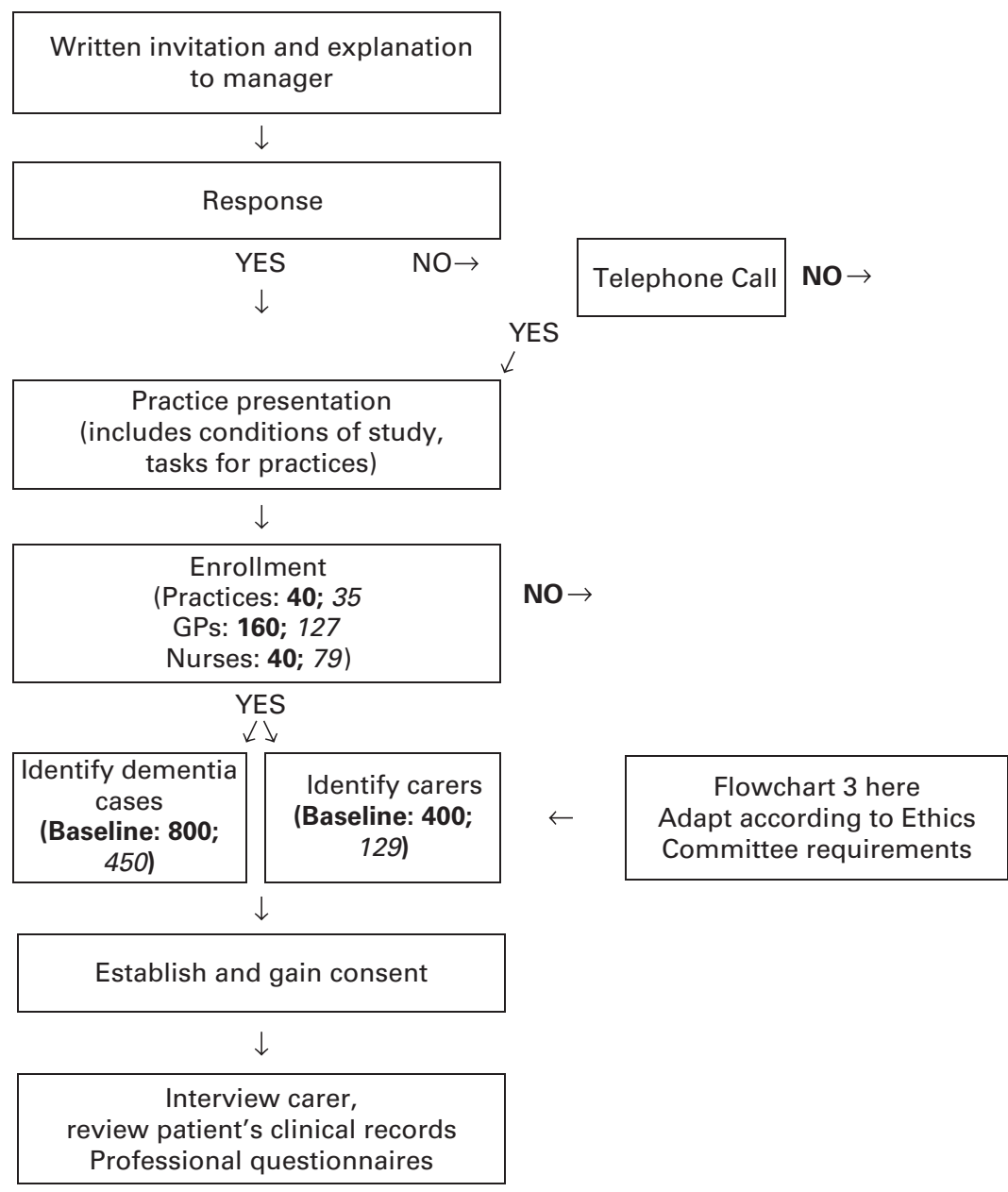

Figure 2 Flowchart of recruitment strategies (Recruitment targets shown in bold, number of valid participants shown in italics)

the two local Primary Care Research Networks, which resulted in several expressions of interest and eventual practice recruitment. The second strategy targeted the Local Health Care Co-operatives (LHCC's) and their primary care locality groups. The heads of all locality groups (and GP's within each group's area) were approached by letter from the study co-ordinator with a request to present the study at their next locality meeting.

After presentation of the study, all practices were contacted by the co-ordinator or research fellow and if interest was expressed, a visit to the practice was made. Over the course of 18 months 20 practices were recruited in this way in Scotland.

\section{Patient and carer recruitment}

Once practices were recruited to the study practice staff had to comply with the study protocol in the recruitment of people with dementia and their carers. The study inclusion and exclusion criteria are shown on Table 2 and a flowchart for identifying eligibility is shown in Figure 3. Attempts were made to simplify this process as much as possible, and to reduce the time required to complete study protocols such as patient identification. Where

Primary Health Care Research and Development 2007; 8: 12-21 
Table 2 Study inclusion and exclusion criteria for patients and carers

\begin{tabular}{|c|c|}
\hline Age & Over 75 \\
\hline Dementia diagnosis & $\begin{array}{l}\text { GP and practice nurse(s) either: strongly suspects has dementia, or has } \\
\text { diagnosed with probable dementia }\end{array}$ \\
\hline Living situation & $\begin{array}{l}\text { In the community with or without carer, and in residential or nursing home } \\
\text { care temporarily in hospital }\end{array}$ \\
\hline In practice & Is currently in the practice list, patient case notes are held by practice \\
\hline Exclusion criteria for & $\begin{array}{l}\text { - If the patient or carer are involved in concurrent research } \\
\text { - If the GP and/or practice nurse(s) feels that an approach to the carer would } \\
\text { be inappropriate, for example the dementia is very severe, or that an } \\
\text { approach may increase distress } \\
\text { - Any other important reason that the GP and practice nurse(s) may have for } \\
\text { why we should not contact the carer, for example they do not know the } \\
\text { probable diagnosis }\end{array}$ \\
\hline
\end{tabular}

Inclusion criteria for carer

Lives with or is in contact at least five times a week (this does not have to be face-to-face contact, eg carer may keep in touch via telephone) with person with dementia. Carer must be aware of probable diagnosis

practically possible research staff assisted with the data collection.

\section{Results}

We identified four major problems in conducting this research (1) multiple obstacles to practice recruitment; (2) the effect of differing Ethics Committee requirements on recruiting patients and carers to the study; (3) practice compliance with patient and carer recruitment processes; and (4) retention of carers, once recruited.

\section{Practice recruitment}

One hundred and twenty seven (127) GPs and 79 nurses enrolled in the study from the 35 practices. There were significant differences in recruitment patterns and outcomes in London and Scotland. The target of twenty practices was met in Scotland before the deadline, but only after extending the recruitment from Forth Valley to Lothian Health Board. Recruitment proved particularly difficult in London, despite a larger practice pool. Despite a variety of approaches and sustained effort, only 16 London practices were recruited. One in four practices approached in Scotland were recruited, but only one in ten in London. A number of factors appeared to affect practice recruitment as shown below.

Primary Health Care Research and Development 2007; 8: 12-21

\section{Time}

Time commitment to the study was the biggest single factor identified by all the practices in deciding whether to participate, particularly the time it would take to go through the lists of patients applying the exclusion criteria.

\section{Relevance}

Not all practices perceived dementia care as a clinical problem warranting priority attention.

\section{Partners}

The project recruited whole practices rather than individual GPs. The GPs interviewed came from a range of practice sizes, from single-handed to practices with up to eight partners. Although full practice agreement was requested it became clear that in some of the larger practices that not all the staff were willing to participate. This led to incomplete sets of questionnaires on professional knowledge, skills and attitudes but did not appear to affect other aspects of the data collection.

\section{Ethics}

Ethical issues were raised by some of the GPs, including concerns about confidentiality, and the impact of research on treatment and care. Ethical committee approval had been gained before approaching practices and most of the GPs concerns raised were covered by this process. Research staff 
Flowchart definitions:

1. Diagnosed: Has patient been diagnosed with dementia, or in GP and/or specialist opinion, has 'probable dementia'.

2. Carer: Family member giving substantial amount of care on at least a weekly basis.

3. Carer/relative (re assent for medical record study): if no carer (as defined above), is there a relative who should be approached for assent?

4. Consent: The patient is, in the GP and/or carer's view aware of diagnosis and capable of understanding the request to check his or her medical records, the undertaking of confidentiality and voluntary nature of consent?

5. Patient Informed: Is the patient aware of diagnosis according to GP, practice team member, other professional or carer?

6. Carer Informed: Is Carer aware of diagnosis according to GP, practice team member or other professional?

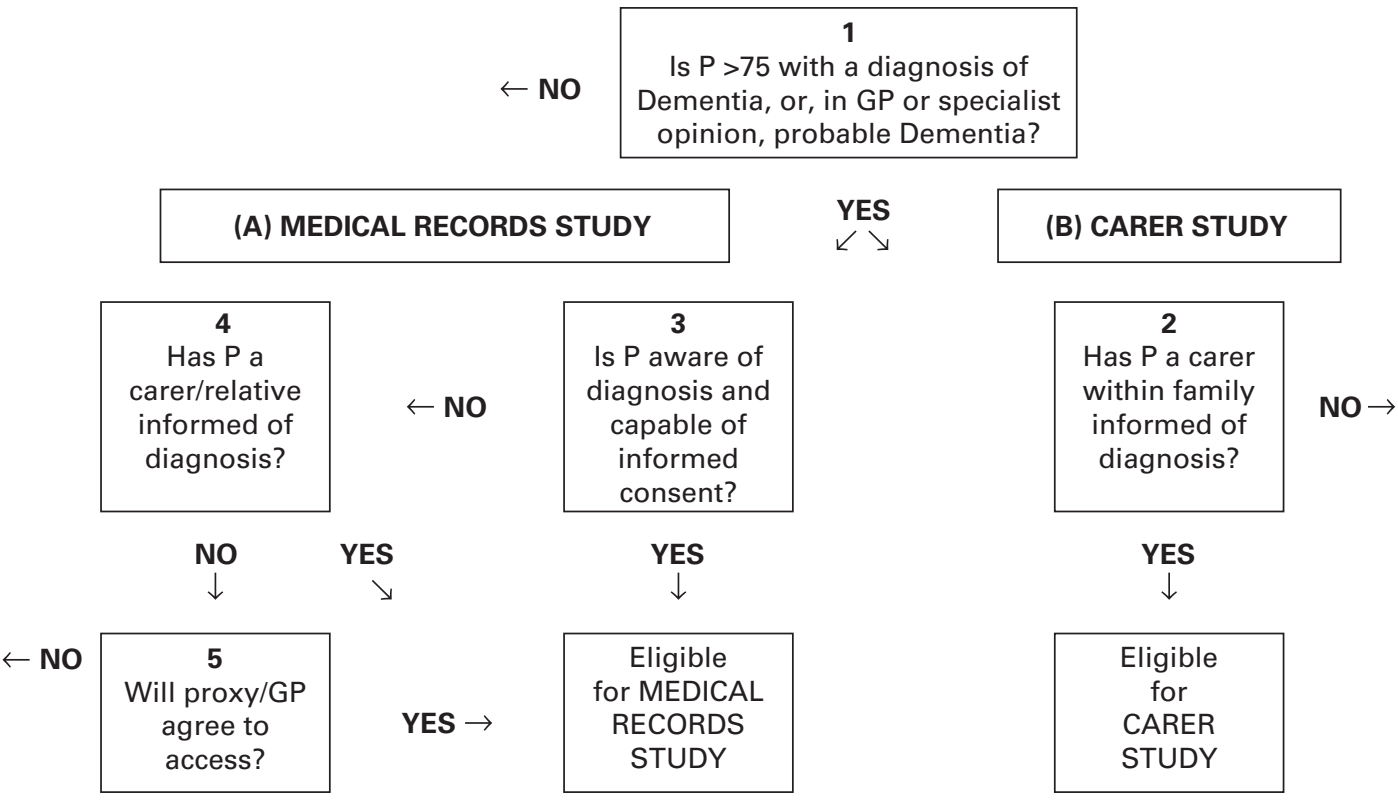

IF 1 = NO: NOT ELIGIBLE FOR EITHER STUDY

IF 2 = NO: NOT ELIGIBLE FOR CARER STUDY

IF 5 = NO: NOT ELIGIBLE FOR MEDICAL RECORDS STUDY

Process of:

If YES to 1-5:

- Initial contact by phone and/or GP letter from practice to carer introducing carer study, asking consent to researcher contact. Practice records numbers involved.

- On contact with carer, interview arranged (CARER STUDY) letter and consent form sent. Research fellow confirms patient ability to give informed consent assessed and patient asked for consent (MEDICAL RECORDS STUDY). Alternatively if carer doubts patient's ability to consent, carer asked for proxy consent.

If YES to all but 3 (no patient informed consent):

- Initial contact by phone and/or GP letter from practice to carer introducing carer study, asking consent to researcher contact. Practice records numbers involved.

- On contact with carer, interview arranged (CARER STUDY) letter and consent form sent. Research fellow confirms patient inability to give informed consent, and asks carer for proxy consent (MEDICAL RECORDS STUDY).

If YES to 1, 3, 4, but NO to 2 (no carer):

- Initial contact by phone and/or GP letter from practice to patient introducing medical records and carer study, asking consent to researcher contact. Practice records numbers involved.

- On contact with patient research fellow confirms no carer and patient ability to give informed consent, and asks patient for consent (MEDICAL RECORDS STUDY).

If YES to 1 only (no carer, no informed consent possible):

- Consent obtained form next of kin or proxy, guided by GP.

Figure 3 Flowchart for identifying subject eligibility 
who did not have a clinical background encountered more resistance to access to patient information than did their clinical counterparts.

\section{Space}

Space was at a premium inside most of the London practices. All enrolled practices were accommodating but delays were experienced with access to a workspace for the research team to search medical records.

\section{Workload}

Anxieties were expressed about additional workload with patients and carers contacting the practice directly regarding the study.

\section{Patient resistance}

In one practice practitioners felt that their patients were well educated and would be resistant to participating in the study, and from this particular practice only one patient and carer agreed to participate out of sixteen eligible patients.

\section{Costs}

The use of locum cover was suggested to the practices in order to provide cover, especially when undertaking training and reimbursement was offered. However, only one practice claimed recompense for such cover.

\section{Research or inspection?}

Inviting in an external team to examine current practice and implement training requires a level of self-confidence on the part of the practice team. Fear of evaluation - which in London involved a Medical School department - may have deterred some practices from participation. In some cases, it was clear that the approach for recruitment to the study had split the practice team, whether between individuals in the same profession or between GPs and nurses.

A deadline for practice recruitment was imposed because of slippage with the study timetable and the time needed for re-interviews of carers, instigating training and completion of evaluations. One London practice was dropped from the study because of lack of completion of the study protocol over a 15-month period: another withdrew after initially agreeing to take part. Four practices were still required to participate and advice was sought from experienced researchers in the locality and a local primary care research network. Selected Primary Health Care Research and Development 2007; 8: 12-21 practices were written to again, with a follow-up phone call, however this did not prove to be fruitful. The result was that the study recruited and retained 35 practices, not the 40 that we had estimated that we would need.

\section{Ethical issues in recruitment}

It was agreed by the three Ethics Committees involved that permission to contact carers and access medical records would be sought from those people with dementia whom the health care practitioner deemed as capable of giving consent. Where the person was not deemed capable, agreement was sought from a family member or patient advocate (see Figure 3 for details). An initial approach to carers was made from the practice, by letter in London and Lothian Health Board, and by telephone in Forth Valley Health Board, before individuals' contact details were passed to the research team. This difference in initial method of contact was dictated by the Ethics Committees in each area, and had implications for recruitment of patients and carers (see Figure 3).

Practices themselves applied rules about access to records very differently. The background of the researchers may have had an impact on recruitment processes, with a more positive response being given to those with a clinical background.

\section{Recruiting patients and carers}

The 35 practices had a combined total of 225740 registered patients, including 13068 aged 75 or over $(5.8 \%)$. This is comparable to a general population figure of $6 \%$ in Scotland and $5.6 \%$ in the relevant districts of London (www.statistics.gov.uk/ census 2001). However, the number of patients aged 75 or over per practice varied greatly, from 34 to 784, with a mean of $373(\mathrm{SD}=210.8)$, as did the proportion of total list size they represented (1.7-11.4\%).

Two major problems arose during the identification of patients and the recruitment of carers: the variable content of electronic medical records, and the lack of documented information about carers in the practices.

\section{Medical records}

The need for practice staff to use computerized medical records in the identification of eligible patients caused problems, even though the use of electronic medical records for routine clinical 
documentation an entry criterion for practice involvement. In some practices records were inadequate in that not all diagnoses or patient information had been recorded electronically. Staff who were initially willing to take part found that when they came to do the search, the information was not readily to hand or that patient records were not up to date. Practices where computer records were used routinely to record diagnoses appeared to recruit patients and carers more easily to the study than those that had to perform hand searches or use recall techniques to identify contact lists.

\section{Identification of patients and carers}

Practices approached identifying eligible patients and carers differently. Some practices did this from recall of their patients, some printed lists of patients aged over 75 on their lists and checked these from recall, others performed an electronic search. Of those who did electronic searches many then had to perform additional hand searches as dementia was not always entered on the electronic record as a coded problem. Particular issues arose when identifying the carers of people with dementia. Practitioners were less likely to recall carers' details and hand searches of the medical record had to be performed.

In both sites, but particularly in London, the process of patient identification proved to be timeconsuming. The average time taken by London practices to identify patients and carers was 15 weeks. This had major implications for the study timetable and resulted in some slippage as research staff had to spend time maintaining frequent contact or making multiple visits to practices to encourage the completion of these tasks. Practices recruited early in the study were no more efficient identifying contact lists or returning paperwork than those recruited later.

Once it was clear that recruitment of patients was slower than expected, it was too late to extend the study to other districts, as Multi-Centre Ethical Committee approval would have been required. Other delays included changes of staff and particularly with the key contact at the practices during the study period. Where this occurred the new key contact had to be briefed about the study and stage of the process by the research staff. This caused considerable delay in completion of the study tasks in these practices.

\section{Involving carers}

Time was repeatedly identified as a barrier to participation by carers. We surmised from speaking with carers who initially agreed to participate but who when contacted to arrange a meeting time were too busy, that they were under considerable strain. The ratio of informal carers to patients was $1: 3$, rather than $1: 2$ as originally estimated. One hundred and twenty nine of the 193 (67\%) carers identified were contacted by the practice and agreed to interview. Two of these carers cancelled appointments, leaving the resulting sample as 127 .

\section{Participants}

Carers had few problems with the interview process. For many the opportunity to speak with someone confidentially about their experiences, needs and fears seemed to be a cathartic and supportive process. The interviews ranged from $45 \mathrm{~min}$ utes to two and a half hours, and no one expressed concern over the length of this process. Most participants were unconcerned at the prospect of the research team looking through their relative's medical records, although reservations were expressed on two occasions and these were respected.

However, attrition of carers at follow-up was high. At follow-up, 52 of the 127 carers were no longer eligible for re-interview: 27 were no longer carers because their relative with dementia had died; 20 people with dementia had left the practice, either because they had gone into residential care or moved closer to relatives; and 5 who consented at baseline were not passed to the study team until follow-up. Of the remaining 75 carers interviewed at baseline, $53(71 \%)$ agreed to be re-interviewed. Practices also identified 59 new carers of patients not identified at baseline: 19 of these refused to be interviewed or could not be contacted, thus yielding an additional sample at follow-up of 40 carers.

\section{Discussion}

Case studies are defined as 'the study of the particularity and complexity of a single case, to understand its activity within important circumstances' (Stake, 1995). What can we learn from this case study of an RCT? We believe there are four important lessons:

1. Epidemiological data yields predictions of dementia prevalence and of the proportions of 
people with dementia with (non-professional) carers that differ from those encountered in selfselected practices joining a research study. Fewer carers were identified than expected, which may be as much a function of the practices' inability to recognize their status or record their details as a demographic peculiarity of an atypical practice sample. This has important implications if carer satisfaction or burden is the primary outcome measure in any future study in a similar setting.

2. Practice recruitment is a problem in primary care research, and more so in dementia research. A number of practices that we approached did not think that a study looking at dementia diagnosis and management was relevant to their work and case mix, a claim that we were unable to verify. However, reports that the practice had few people with dementia on their patient lists may reflect the problem of under-recognition. Recruitment may have been biased towards practices already interested in the subject matter, or in research and education. Economic incentives did not appear to have a powerful effect on recruitment, confirming Jonker and Sumajow's view from a decade earlier (Jonker and Sumajow, 1992).

3. Once recruited, in most cases there were problems adhering to the research protocol. There were long delays in some practices in carrying out some tasks especially those related to the identification of eligible patients and carers. Tognoni et al. (1991), found similar problems with compliance even if the GPs themselves were involved in developing the original protocol. The quality of electronic medical records is very variable, and identification of both diagnoses and carer status was problematic.

4. Carers may not feel able to sustain an involvement in research because of the nature of the caring experience. However, research encounters may have some supportive benefit. The requirements of Ethics Committees added to the complexity of the research task and so contributed to underperformance in the practices.

\section{Conclusion}

The messages for researchers seeking to undertake dementia-related trials in primary care are stark. Recruitment of general practices to any Primary Health Care Research and Development 2007; 8: 12-21 study of dementia care will be challenging due to the nature of the work environment. This is particularly the case where the study involves considerable numbers of practices, where there is a perception of burden, or where the subject matter is not among the highest concerns identified by the team. Practices may express ambivalent attitudes to research, seeing it as 'the right thing to do' yet not committing time and resources to projects even when external funding is made available. This is more likely to occur when historically high levels of demand and problems of staffing and organization destabilize practices (as in the London site) or when differences of opinion in the practice lead to variable commitment by staff.

More work has to be done to encourage research to take place both within, and by, primary care (Mant, 1997) but specific strategies for doing this are not yet obvious. Our experience supports the view that maintaining regular and personal contact with practices appears to have some effect. The experience of this study in Scotland would indicate that using the primary care hierarchy to reach large groups appears to be helpful. Defining a particular person within a practice and building up a relationship over time, and making research tasks explicit also appears to aid completion of study protocols. Researchers should not underestimate the problems that they will face, even in a carefully designed study, and funders should be aware of the implications for study length and resourcing.

\section{Acknowledgements}

The authors would like to thank the Alzheimer's Society (Alexander and Christina Dykes Project Grant) for their funding of the research study 'Dementia: Diagnosis and Management in Primary Care'. The carers, people with dementia, practices and staff who participated in the study for their time and valuable contribution. We would also like to thank Ms Deborah Haworth for her contribution to the fieldwork, and Forth Valley and Lothian Primary Care Research Networks for help in piloting and practice recruitment.

\section{References}

Abbasi, K. 1999: Guidelines for evaluating papers on educational interventions British Medical Journal 318, 1265-67. 
Audit Commission. 2000: Forget me not: Developing mental health services for older people in England. London: Audit Commission.

Audit Commission Update. 2002: Forget me not: Developing mental health services for older people in England. London: Audit Commission.

Boise, L., Camicioli, R., Morgan, D.L., Rose, J.H. and Congleton, L. 1999: Diagnosing dementia: perspectives of primary care physicians. Gerontologist 39, 457-64.

Cochrane, A.L. 1972: Effectiveness and efficiency: random reflections on health services. London: Oxford University Press.

Department of Health. 2005: Briefing document for applications for local research networks for neurodegenerative disorders and dementias. Department of Health, London.

Downs, M. 1996: The role of general practice and the primary care team in dementia diagnosis and management. International Journal of Geriatric Psychiatry 11, 937-42.

Downs, M., Turner, S., Iliffe, S., Bryans, M., Wilcock, J., Keady, J., Levin, E., O'Carroll, R.E. and Howie, K. 2006: Effectiveness of educational interventions in improving detection and management of dementia in primary care: a cluster randomized controlled study. British Medical Journal 332, 692-96.

Fortinsky, R.H., Leighton, A. and Wasson, J.H. 1995: Primary care physicians' diagnostic, management, and referral practices for older persons and families affected by dementia. Research on Ageing 17, 124-48.

Haines, A. and Iliffe, S. 1995: Innovations in service and the appliance of science. British Medical Journal 310, 815-16.

Iliffe, S. 1997: Can delays in the recognition of dementia in primary care be avoided? Ageing and Mental Health 1, 7-10.

Iliffe, S., Wilcock, J., Downs, M., Turner, S. and Bryans, M. 2002a: A randomised controlled trial of educational interventions for dementia diagnosis and management in primary care. Neurobiology of Aging 23(Suppl 1), S550-51.

Iliffe, S., Wilcock, J., Austin, T., Walters, K., Rait, G., Turner, S., Bryans, M. and Downs, M. 2002b: Dementia diagnosis and management in primary care: developing and testing educational models. Dementia 1,11-23.

Jonker, P.L. and Sumajow, C. 1992: Randomised clinical trials in general practice. British Medical Journal 304, 508.

Mant, D. 1997: $R$ \& D in Primary Care. London HMSO, 1997. (National Working Group Report, November 1997).

Stake, R. 1995: The Art of Case Study Research. California: Sage Publications, p. xi.

Thomas, P. 2000: The research needs of primary care. British Medical Journal 321, 2-3.

Tognoni, G., Alli, C., Aranzini, F., Bettelli, G. and Colombo, F. 1991: Randomised clinical trials in general practice: lessons from a failure. British Medical Journal 303, 969-71.

Turner, S., Iliffe, S., Downs, M., Bryans, M., Wilcock, J. and Austin, T. 2003: Decision support software for dementia diagnosis and management in primary care: relevance and potential. Aging \& Mental Health 7, 28-33.

Wilcock, J., Iliffe, S., Walters, K., Rait, G., Austin, T., Turner, S., Bryans, M., Downs, M., Levin, E., O'Carroll, R. and Keady, J. 2002: The development of an evidence-based curriculum for dementia care training in general practice. Education \& Ageing 17, 217-36.

Wilson, S., Delaney, B.C., Roalfe, A., Roberts, L., Redman, V., Wearn, A.M. et al. 2000: Randomised controlled trials in primary care: case study. British Medical Journal 321, 24-27.

Woods, R., Moniz-Cook, E., Iliffe, S., Campion, P., Vernooij-Dassen, M., Zanetti, O. and Franco, M. 2003: Dementia: issues in early recognition and intervention in primary care. Journal of the Royal Society of Medicine 96, 320-24. 